\title{
WISE AND AKARI
}

\author{
ANDREW W. Blain \\ X-Ray and Observational Astronomy, Physics and Astronomy, University of Leicester, Leicester, UK, LE1 7RH \\ E-mail:ab520@leicester.ac.uk \\ (Received June 28, 2012; Accepted July 30, 2012)
}

\begin{abstract}
The first all-sky mid-/far-infrared survey by IRAS in the 1980s, has been followed by only two more, by AKARI, from 2006, and WISE in 2010. I discuss some features of the WISE survey, and highlight some key results from early extragalactic observations that have been made by the science team during the operation of the telescope, and the post-operation proprietary period during which the public release data products were being generated. The efficient survey strategy and very high-data rate from WISE produced a catalogue of 530 million objects that was released to the public in March 2012. The WISE survey strategy naturally provided the deepest coverage at the ecliptic poles, where matched comparison fields were obtained using Spitzer, and where AKARI also observed deep fields. I describe some of the follow-up work that has been carried out based on the WISE survey, and the prospects for enhancing the WISE data by combining the AKARI survey results are also discussed. While the all-sky AKARI survey is less deep than the WISE catalogue, and is still being worked on by the AKARI science team, it includes a larger number of bands, extends to longer wavelengths, and in particular has very complementary band passes to WISE in the mid-infrared waveband, which will provide enhanced spectral information for relatively bright targets.
\end{abstract}

Key words: galaxies: active; galaxies: formation; galaxies: photometry; quasars: general; galaxies: starburst; surveys

\section{INTRODUCTION}

The Wide-Field Infrared Survey Explorer (WISE) is a NASA MIDEX all-sky infrared survey mission that launched in December 2009, and operated for over a year, returning data for two complete mappings of the sky (Wright et al., 2010). Orbiting above the terminator, and always pointing directly away from the Earth, each of the spacecraft's $\simeq 90$-minute orbits scanned the instruments along $\mathrm{a} \simeq 48$-arcmin-wide swath on the sky, producing a great circle scan that included both the north and south ecliptic poles. The survey swaths from adjacent orbits were offset by about 4 arcmin at the equator, and thus the sky was sampling in a series of overlapping circles, leading to a complete coverage of the sky after six months. Modest adjustments to the scan direction was necessary each month to ensure that the moon did not lie within the surveyed swath. The first sky coverage was completed on 17 July 2010, and the final release of the all-sky data products was made on 14th March $2012^{1}$. A preliminary data release, covering $55 \%$ of the sky from the first half of the survey in early 2010 was made in spring 2011, and the final data release took place on 14th March 2012. An image atlas, and a 530-million-source catalogue are the main products, along with a reject catalogue of less-reliable objects.

In addition, NEOWISE, an extension to the original programme to search for Near Earth Objects (Mainzer et al., 2011) was supported by NASA's Planetary Sciences Division, and provided the resources to reduce

\footnotetext{
1 https://ceres.ipac.caltech.edu.
} 
and distribute images from each individual WISE image frame that was taken, from which the moving tracks of asteroids and comets could be selected and recovered.

WISE used a scan-mirror to lock the image from a smoothly-scanning 40-cm aperture cryogenic telescope on to four $1024^{2}$ detectors, simultaneously observing a series of square $48 \times 48-\operatorname{arcmin}^{2}$ frames through dichroics in bands centered at 3.3, 4.7, 12 and $22 \mu \mathrm{m}$, labelled W1-4. The W1 and W2 bands are close to the Spitzer IRAC bands 1 and 2, the broad W3/12- $\mu \mathrm{m}$ band is similar to the corresponding band flown aboard IRAS, and the W4 band is a slighter bluer match to the Spitzer MIPS 24- $\mu \mathrm{m}$ band. In comparison with the AKARI survey, all the detectors aboard WISE were read out in each pointing, and thus WISE produced a much greater data rate, which explains the significantly greater depth of the WISE survey.

The interface between the W1 and W2 bands was carefully designed to ensure that methane absorption in the atmospheres of cool stars and brown dwarfs would lie within $\mathrm{W} 1$ alone, producing very red colors in [W1 - W2], allowing these sources to be highlighted cleanly. The lack of an optical counterpart would then motivate follow-up observations to confirm a large sample of brown dwarves (e.g., Kirkpatrick et al., 2011).

In August 2010, after about seven months of survey operations, and a complete coverage of the sky in all four bands, the primary solid-hydrogen coolant aboard WISE began to run out, ending the flow of useful data from the W3 and W4 channels. For the remainder of the mission, and for an additional period of overlap sufficient to ensure a double encounter with any objects orbiting in the asteroid belt, WISE returned images in the W1 and W2 bands until the end of January 2011.

The exposure time in each frame was matched to the spacecraft's scan rate, at 8 seconds, with an additional 3 seconds for the the scan mirror to return to its started place so data taking could begin for the next tile. Adjacent tiles along the scan swath have a modest overlap region. The typical number of tiles that cover any point of the sky is least on the equator - at about 15 visits - and has a steadily increasing distribution towards the poles, with a maximum coverage of many hundreds of visits within a 5-square-degree area at the ecliptic poles (Jarrett et al., 2011). The significant number of visits, all separated by about 90 minutes, and experiencing different radiation and scattered-light environments, ensured that excellent artifact removal and re- liability were obtained. The data processing approach is built upon the legacy of the all-sky 2MASS survey (Cutri et al., 2012).

The cadence of visits is unusual, with about $5 \%$ of objects being imaged on the opposite edges of adjacent tiles, thus having two 8 second exposures available, with a 3 -second gap between them, and thus being sensitive to variability on a 10 -second timescale. Almost every point on the sky was covered once every $\sim 90 \mathrm{~min}$ orbit over a period of about a day, leading to typically fifteen or more exposures, and then a similar set of observations made six months later. Thus there is variability information available for all points on the sky on a timescale from 90-minutes to one day, and for some on both a 6 -month timescale and a 10-second timescale. The availability of this variability information for other scientific exploitation (e.g., for an X-ray binary in Gandhi et al., 2011) is enabled by the additional support of the NEOWISE project.

WISE's key science goals were the detection of the nearest star, and the most luminous galaxy (and all their slightly less extreme cousins). The all-sky coverage, and excellent mid-infrared sensitivity (Wright et al., 2010) has probed this range, from brown dwarves (Kirkpatrick et al., 2011) to extreme mid-infraredselected ultraluminous galaxies (ULIRGs) and AGN to high-redshift (Eisenhardt et al., 2012; Wu et al., 2012; Bridge et al., 2012).

I will now describe some of the science areas that have been addressed using WISE, from a personal perspective, and then move on to discuss some of the synergies with the forthcoming AKARI products.

\section{EXTRAGALACTIC SCIENCE WITH WISE}

The WISE science team had a proprietary period that extended from the receipt of data at the data center to the public data releases, during which to find and follow-up interesting detected objects. The legacy of IRAS demonstrates that the number of publications flowing from this type of survey is expected to remain almost constant with time, and so the final legacy of the successfully-delivered products from WISE is sure to grow to far exceed what the science team has been able to demonstrate to date. I will now highlight some of the results of the follow-up that has been carried out by the extragalactic fraction of the WISE science team and their collaborators during the period from the acquisition of the first data in early 2010 . 
The process of selecting interesting objects to study in the WISE data has been informed by knowledge gained using IRAS and Spitzer and from ground-based (sub)mm-wave telescopes. However, the all-sky nature of the WISE survey makes it difficult to connect directly the properties of WISE galaxies with those identified and studied in the rich legacy of Spitzer (Soifer, Helou \& Werner, 2008). While even a minute-long observation with Spitzer is deeper than the WISE catalogue, the instantaneous field of view of WISE is approximately 100 times larger, and WISE observed very efficiently throughout its finite lifetime. Thus, extrapolation of Spitzer results is necessary, to compare with both rarer and brighter objects in WISE. Direct comparisons between the WISE ecliptic pole fields (Jarrett et al., 2011) and the properties of AGNs in COSMOS (Stern et al., 2012) have validated like-for-like WISESpitzer comparisons. Statistical properties of the populations of objects throughout the WISE survey, for example by cross-correlation against SDSS are also underway (Donoso et al., 2012; Yan et al., 2012).

\subsection{WISE-Based Selection Techniques}

A variety of approaches have been taken to highlight objects for follow-up investigation. The majority of objects found in the WISE catalogue have stellar colors; both individual galactic stars, and galaxies with evolved stellar populations and little emission from their interstellar medium (ISM). The most useful colorcolor diagram for the discrimination between different populations of objects is obtained by plotting [W1 W2] against [W2 - W3] (see Wright et al., 2010; Jarrett et al., 2011; Stern et al., 2012) ${ }^{2}$. The longestwavelength $\mathrm{W} 4$ band has been used to highlight powerful dust-emitting galaxies; however, the images in the W3 band are about a factor of ten deeper than W4, and so for the bulk of galaxy selections, the three shorter bands are used, followed by a check of the properties of the highlighted sources in the W4 band. In both [W1 - W2] and [W2 - W3] colors, stars cluster around zero, while different classes of galaxies extend to [W2 - W3] $\simeq 7$ and $[\mathrm{W} 1-\mathrm{W} 2] \simeq 2$. SDSS spectral catalogues were correlated with the WISE products to highlight regions of the color-color space where different popula-

\footnotetext{
2 A color-color figure is not shown in this brief review that intends to highlight sources of information about WISE. These references and the release notes (http://wise2.ipac.caltech.edu/docs/release/allsky/) provide a much more complete picture of the results of the survey.
}

tions were found.

A concern before the mission was that cool, dusty AGB stars could have red colors similar to extragalactic objects of interest, but by imposing a requirement that candidates for follow-up were optically faint in the SDSS and DPOSS surveys, the contamination rate from stars was very small. Galaxies containing both powerful AGNs and active star formation produce redder colors in the longer-wavelength $\mathrm{W} 3$ and $\mathrm{W} 4$ bands, and dust obscuration also reddens the emission in [W1 - W2]. Extremely red colors in [W2 - W3] are found, corresponding to the Wien side of the hot dust spectrum, and in some cases, to additional absorption by an optically thick ISM in front of the underlying hot dust (Wu et al., 2012).

The prime technique for identification is on the grounds of red colors in both [W1 - W2] and [W2 W3]. A primarily W3-selected sample, with either faint detections or upper limits in $\mathrm{W} 1$ and $\mathrm{W} 2$ has been used to define a population of more than 1,000 'W1W2 dropouts' (Eisenhardt et al., 2012). This has proved to be a reliable way to select WISE ULIRGs; spectroscopic follow up reveals a rich range of interacting ULIRGs and AGNs from redshifts $z \simeq 0.1$ to $z>4$.

The WISE beam-size is 6 arcsec across, which might suggest a challenge for targeting spectroscopic observations in the absence of an optical or radio counterpart. However, the large size of the WISE tiles (48 arcmin), and the stability of the space-borne platform ensures that the relative positions of the centroids of the targets and guide stars are known to sub-arcsecond precision. This permits reliable spectroscopy without the need for pre-imaging.

By imposing additional color cuts, it is also possible to highlight blue compact dwarf (BCD) galaxies with high specific star-formation rates and low-metallicities in the local Universe (Griffith et al., 2011). These have similar WISE colors, and can be observed efficiently in the same way as the high-redshift samples.

WISE color selection can also reveal massive evolved galaxies at high redshifts, which have a characteristic redshifted red stellar population. The Massive Distant Clusters of WISE Survey (MaDCoWS; Gettings et al., 2012) is a concerted follow-up campaign to find highredshift clusters of galaxies by identifying spatial overdensities of galaxies with consistently red stellar populations in the WISE catalogue, and then following-up the brightest candidates spectroscopically to confirm 
their redshifts (e.g., Stanford et al., 2012). There are excellent synergies with the all-sky survey for SunyaevZeldovich signatures of clusters made using the Planck Surveyor mission. The unprecedented depth and sky coverage available from WISE, has made it possible to increase the number of high-redshift cluster candidates by a significant factor.

\subsection{WISE Properties of Known Samples}

The properties of any existing classes of galaxies in the WISE survey, regardless of their distribution on the sky, provides new insight into the overall properties of galaxies, and an additional way to measure the total bolometric luminosity of samples of galaxies with previously sparse mid-infrared data. Two of the most dramatic examples are samples of galaxies that were selected by combining WISE data with the results of the NVSS/FIRST radio surveys and serendipitous hard $\mathrm{X}$-ray surveys.

Wide-field radio surveys have been cross-correlated with WISE by Lonsdale et al. (2011), to produce a set of radio-intermediate galaxies at moderate and high redshifts, which are much brighter in the radio than the radio-far-infrared correlation would suggest, but which are not as luminous in the radio as classical double radio galaxies. The expectation is that this technique selects powerful AGN jets that are interacting strongly with the ISM of their hosts, an idea that is being tested using interferometric observations with eVLA/Jansky array and ALMA. An insight into some of the astrophysics involved in these feedback processes are discussed by Farrah et al. (2012). These WISE-radio galaxies could be a rare population caught in the act of having their luminosity influenced by AGN power, and could potentially provide a snapshot of some of the most intense feedback activity in the Universe. Without the WISE information over wide fields, it would have been impossible to recognize these galaxies within the large radio samples.

The BUXS hard X-ray sample of 258 galaxies (Mateos et al., 2012) is almost spectroscopically complete, and was selected from X-ray properties in the large deep 2XMM survey. 2XMM is the largest compiled sample of X-ray selected galaxies, constructed from all the data that has been obtained from the XMMNewton mission (Watson et al., 2010). From the WISE colors of this large sample, it was possible to generate their spectral energy distributions (SEDs), and a reli- able WISE-color-selection region that highlights AGN, and provides the opportunity to derive an efficient allsky sample of WISE-selected AGN, for which X-ray data is not typically available.

There are other examples of efforts to exploit the WISE data to investigate previously known classes of galaxies with little mid-infrared data. These include far-infrared-selected galaxies from the WISE counterparts of Herschel-ATLAS sources, detected in the largest survey during the Herschel mission (Bond et al., 2012), for the increasingly large survey areas of the South Pole Telescope (SPT) survey, high-redshift AGN (Blain et al., 2012) and for UV-selected galaxies from GALEX (Petty et al., 2012). The large scale structure of galaxies traced by the $2 \mathrm{dF}$, SDSS and $6 \mathrm{dF}$ galaxy redshift surveys has also been investigated in the context of their WISE properties.

\subsection{Details of WISE Follow-up Observations}

Spectroscopic surveys for WISE dropouts using 4- and 8-10-m class telescopes have enabled more detailed and comprehensive follow-up investigations using a wide range of multiwavelength facilities, many of which are still ongoing.

In many nights of spectroscopy, a wide variety of galaxy types are revealed, from broad-line AGN, including examples with unusual absorption in the broad lines, to star-forming galaxies indistinguishable from Lyman-break galaxies (Lake et al., 2012), and objects with strong extended Lyman- $\alpha$ emission. Bridge et al. (2012) describe a sample of Lyman- $\alpha$ blobs (LABs), including the first identified WISE ULIRG W1814+34 (Eisenhardt et al., 2012), that were found to have extended emission, revealing a large Lyman- $\alpha$ halo around these objects. By modifying the WISE color selection criteria slightly in response to these discoveries, it is possible to obtain samples of tens of these LABs, which prior to WISE had been found in only small numbers, many from Subaru wide-field narrowband surveys.

Investigations of WISE-selected sources include a series of over 1,000 warm Spitzer observations to measure accurate colors in the equivalent $\mathrm{W} 1$ and $\mathrm{W} 2$ bands using the much more sensitive Spitzer IRAC camera (along with a brown dwarf candidate Spitzer program), ground-based AO near-infrared imaging to resolve interesting candidates, and optical imaging to determine morphologies and colors that are not available in exist- 
ing wide-field catalogues. In 2012 several WISE-based proposals were accepted for high-resolution imaging using Hubble Space Telescope.

At longer wavelengths, comprehensive far-infrared SEDs are being found for a hundred W1W2 dropouts using Herschel, and there are measurements and limits of the mm-wave dust continuum flux density for several tens of examples using an array of available groundbased (sub-)mm-wave telescopes, including ALMA, ATCA, IRAM, CSO and JCMT. The main result of this long-wavelength investigation is that the WISEselected galaxies are ultraluminous, but display significantly hotter bulk dust temperatures than existing samples of far-infrared selected galaxies (Wu et al., 2012), and have relatively faint CO molecular gas emission. This has the consequence that substantial (sub)mm-wave surveys of the properties of these galaxies require the power of ALMA, and are difficult using smaller facilities. There are also excellent prospects for obtaining radio continuum imaging of WISE-selected galaxies using the eVLA/Jansky array; the NVSS/WISE sample is currently being pursued in this way. The advent of next-generation arrays like LOFAR, MeerKAT and ASKAP will provide the opportunities to observe the radio emission from these very-luminous galaxies.

\section{WISE AND UNDERSTANDING GALAXY EVOLUTION}

The most luminous objects during the evolution of the Universe are being highlighted using WISE (Eisenhardt et al., 2012). The WISE-selected samples that have been found by the science team should be very useful for addressing a variety of problems in understanding galaxy evolution. In particular, the discovery of new ULIRGs (Eisenhardt et al., 2012; Wu et al., 2012), extended LABs (Bridge et al., 2012), and radio-WISE selected AGNs (Lonsdale et al., 2011), highlight new samples of galaxies that appear to be interacting strongly with their environments, and yet which have been very difficult to recognize in the absence of mid-infrared data. They are ultraluminous, and so are known to be powered by very intense activity, and are likely to be experiencing the strong feedback effects that are often assumed to be responsible for establishing the properties of galaxies today. By exploiting the spectral resolving power of powerful instruments like ground-based 8-m-class AO-fed near-infrared telescopes, ALMA, the
eVLA/Janksy array, and JWST, it will be possible to reveal the motions and excitation of the gas within, and to investigate feedback processes in galaxies with unprecedented luminosities all over the sky.

\section{EXPLOITING WISE AND AKARI TOGETHER}

The existence of the all-sky catalogue from AKARI, substantially deeper than IRAS and with greater resolution than Planck Surveyor, will provide significant opportunities to extend the exploitation of the WISE catalogue into the far-infrared waveband. While there will be Herschel follow-up observations for hundreds of WISE-selected galaxies at wavelengths from 70 $500 \mu \mathrm{m}$, and wide-field Herschel surveys cover up to $1,000 \operatorname{deg}^{2}$ as a legacy, there is no substantial capability for extending the far-infrared coverage of specific objects until SPICA is in service. A clear understanding of the populations of bright far-infrared galaxies will doubtless help in planning spectroscopic campaigns using that facility.

As additional generations of the AKARI catalogue are produced, the availability of the WISE catalog as a truth table should assist in the quality control and testing of the AKARI data, allowing the reliable extrapolation of the SEDs of WISE detections at W4 out to longer far-infrared wavelengths. For moderate-redshift galaxies, the availability of this resource will also help significantly with the planning of observations using JWST.

In particular, note that the WISE W3 $12-\mu \mathrm{m}$ filter is very broad, and so spectral features within - including PAH structure and silicate absorption features - cannot be resolved easily. By combining the images in W3 and the bracketing AKARI bands (S9W and L18W), a color within this band can be extracted for WISE-AKARIdetected galaxies. For many of the galaxies that are clearly detected by WISE in the W4 band, this will provide a valuable extension of our knowledge of their full mid-infrared SEDs.

\section{CONCLUSIONS}

WISE has produced a new all-sky infrared survey in four bands from $3-22 \mu \mathrm{m}$. The colours of detected galaxies have been used to highlight interesting cases for detailed follow-up observations, and have highlighted large samples of previously rare or hard-tofind galaxies, including dusty ULIRGs with hot SEDs, 
Lyman- $\alpha$ blobs (LABs), a mid-infrared variable galactic blackhole, and radio-detected galaxies that appear to be involved in violent feedback.

The public WISE survey should provide a valuable reference for comparison with the AKARI all-sky survey products, while the availability of AKARI data at both longer wavelengths and in filters that are complementary to the WISE bands will provide significantly more information about the mid-infrared SEDs of the brighter objects in the WISE catalogue.

\section{ACKNOWLEDGEMENTS}

The author acknowledges the efforts of the WISE science and project teams who enabled and conducted the work described here, and a Royal Society Wolfson Research Merit Award. This publication makes use of data products from the Wide-field Infrared Survey Explorer (WISE), which is a joint project of the University of California, Los Angeles, and the Jet Propulsion Laboratory/California Institute of Technology, funded by the National Aeronautics and Space Administration.

\section{REFERENCES}

Blain, A. W., et al., 2012, WISE Observations of $z>$ 6 AGNs, ApJ, submitted

Bond, N. A., et al., 2012, The Infrared Properties of Sources Matched in the WISE All-sky and Herschel ATLAS Surveys, ApJ, 750, L18

Bridge, C., et al., 2012, A New Population of High Redshift, Dusty Lyman-Alpha Emitters and Blobs Discovered by WISE, ApJ, submitted, arXiv: 1205.0385

Cutri, R. M., et al., 2012, Explanatory Supplement to the WISE All-Sky Data Release Products, http://wise2.ipac.caltech.edu/docs/release/ allsky/expsup/index.html

Donoso, E., et al., 2012, Origin of 12- $\mu \mathrm{m}$ Emission across Galaxy Populations from WISE and SDSS Surveys, ApJ, 748, 80

Eisenhardt, P., et al., 2012, The First HyperLuminous Infrared Galaxy Discovered by WISE, ApJ, 755, 173

Farrah, D., et al., 2012, Direct Evidence for Termination of Obscured Star Formation by Radiatively Driven Outflows in Reddened QSOs, ApJ, 745, 178

Gandhi, P., et al., 2011, A Variable Mid-infrared Synchrotron Break Associated with the Compact Jet in GX 339-4, ApJ, 740, L13
Gettings, D. P., et al., 2012, Massive Distant Clusters of WISE Survey. I. The First Distant Galaxy Cluster Discovered by WISE, ApJ, in press, arXiv: 1205.7092

Griffith, R. L., et al., 2011, WISE Discovery of Lowmetallicity Blue Compact Dwarf Galaxies, ApJ, 736, L22

Jarrett, T., et al., 2011, The Spitzer-WISE Survey of the Ecliptic Poles, ApJ, 735, 112

Kirkpatrick, J. D., et al., 2011, The First Hundred Brown Dwarfs Discovered by the Wide-field Infrared Survey Explorer (WISE), ApJS, 197, 19

Lake, S. E., et al., 2012, Optical Spectroscopic Survey of High-latitude WISE-selected Sources, AJ, 143, 7

Lonsdale, C. J., et al., 2011, Red Radio-intermediate Quasars From The Wise Survey, BAAS, 21832711

Mainzer, A., et al., 2011, Preliminary Results from NEOWISE: An Enhancement to the Wide-field Infrared Survey Explorer for Solar System Science, ApJ, 731, 53

Mateos, S., et al., 2012, Using the Bright Ultra-Hard XMM-Newton Survey to Define a MIR AGN Selection Based on WISE Colours, MNRAS, in press, arXiv:1208:2530

Petty, S., et al., 2012, The Origin of the Ultraviolet Excess: Evidence for a Multistage Formation History of Local Early Type Galaxies using WISE and GALEX, ApJ, submitted

Soifer, B. T., Helou, G., \& Werner, M., 2008, The Spitzer View of the Extragalactic Universe, ARA\&A, 46, 201

Stanford, S. A., et al., 2012, IDCS J1426+3508: Discovery of a Massive, IR-Selected Galaxy Cluster at $z=1.75, \mathrm{ApJ}, 753,164$

Stern, D., et al., 2012, Mid-infrared Selection of Active Galactic Nuclei with the Wide-Field Infrared Survey Explorer. I. Characterizing WISE-selected Active Galactic Nuclei in COSMOS, ApJ, 753, 30

Watson, M. G., et al., 2010, The XMM-Newton Serendipitous Survey. V. The Second XMMNewton Serendipitous Source Catalogue, A\&A, 493, 339

Wright, E. L., et al., 2010, The Wide-Field Infrared Survey Explorer (WISE): Mission Description and Initial On-orbit Performance, AJ, 140, 1868

Wu, J. -W., et al., 2012, Submillimeter Follow-up of WISE-Selected Hyperluminous Galaxies, ApJ, 756,96 
Yan, L., et al., 2012, Characterizing Mid-Infrared Extragalactic Sources Revealed by the Wide-Field Infrared Survey Explorer (WISE), ApJ, submitted 